\title{
Features of Nonlocal Chiral Quark Models
}

\author{
D. Gómez Dumm \\ IFLP, CONICET - Dpto. de Física, Universidad Nacional de La Plata, C.C. 67, 1900 La Plata, Argentina \\ CONICET, Rivadavia 1917, 1033 Buenos Aires, Argentina
}

(Received on 5 July, 2008)

\begin{abstract}
Main features of nonlocal chiral quark models are discussed, focusing on the description of low energy phenomenology and the analysis of phase transitions at finite temperature and/or chemical potential. Applications to compact stars are commented.
\end{abstract}

Keywords: Chiral quark models; QCD phase transitions; Quark matter

\section{INTRODUCTION}

The description of strong interactions in the nonperturbative regime is still one of the most important open problems in particle physics. In particular, the detailed knowledge of the phase diagram for strongly interacting matter has become an issue of great interest in recent years, in view of the development of the experimental programs with ultrarelativistic heavy ion beams at CERN-SPS and BNL-RHIC and the applications of this subject to cosmology and astrophysics. On the theoretical side, even if a significant progress has been made on the development of ab initio calculations such as lattice QCD $[1,2]$, these are not yet able to provide a full understanding of the QCD phase diagram due to the well-known difficulties of dealing with finite chemical potentials. Therefore, it is important to develop effective models that show consistency with lattice results and can be extrapolated into regions not accessible by lattice calculation techniques.

One of the most popular approaches to an effective description of the QCD interactions is the quark version of the Nambu-Jona-Lasinio (NJL) model [3, 4], in which quark fields interact through local four point vertices that satisfy chiral symmetry constraints. As an improvement of this simple approach, we consider here chiral quark models that include nonlocal covariant interactions. In fact, nonlocality arises naturally in the context of several successful approaches to lowenergy quark dynamics, as e.g. the instanton liquid model [5] and the Schwinger-Dyson resummation techniques [6]. This is in agreement with lattice QCD calculations [7], which also indicate that quark interactions should act over a certain range in momentum space. Moreover, it has been shown that nonlocal covariant extensions of the NJL model have some advantages over the local theory, like e.g. a natural regularization scheme in which anomalies are preserved [8] and next-toleading order corrections are found to be relatively small [9]. In what follows we will present an overview of several works carried out on this subject [10-18], studying phenomenological features of nonlocal chiral quark models and analyzing within this context the structure of the phase diagram of strongly interacting matter. In order to include a description of the confinement/deconfinement transition we will also consider a further extension of these models in which quarks are coupled with the Polyakov loop.

The article is organized as follows. In Sect. II we introduce the models, considering two possible schemes to account for the nonlocality, and we show the theoretical con- sistency between these schemes and well-known chiral relations. In Sect. III we present the numerical results obtained for the model parameters for definite nonlocal form factors, discussing the corresponding phenomenological implications. Then, in Sect. IV we describe the $T-\mu$ phase diagrams obtained for isospin symmetric quark matter and for quark matter that satisfies compact star conditions of neutrality and $\beta$ equilibrium. The application of these nonlocal models to the description of compact star cores is also discussed. In Sect. V we conclude our overview by describing some features of nonlocal models in which quarks are coupled with the Polyakov loop. Finally, Sect. VI includes a summary of this presentation.

\section{NONLOCAL TWO-FLAVOR CHIRAL QUARK MODELS}

\section{A. Euclidean action}

We begin by stating the Euclidean action for a two-flavor nonlocal chiral quark model that includes a four point quarkantiquark interaction:

$$
S_{E}=\int d^{4} x\left\{\bar{\psi}(x)(-i \partial+\hat{m}) \psi(x)-\frac{G_{S}}{2} j_{M}^{a}(x) j_{M}^{a}(x)\right\} .
$$

Here $\psi \equiv(u d)^{T}$ is a fermion doublet and $\hat{m}=\operatorname{diag}\left(m_{u}, m_{d}\right)$ stands for the current quark mass. For simplicity we will work in the isospin limit, in which $m_{u}=m_{d}=\bar{m}$. The nonlocality of the quark-antiquark currents $j_{M}^{a}(x)$ can be introduced in different ways [19]. Here we will consider two scenarios that we call "Scheme I" and "Scheme II". In the case of Scheme I [20, 21], the effective interactions are based on an instanton liquid picture of QCD [5]. The currents can be written as

$$
j_{M}^{a}(x)=\int d^{4} y d^{4} z r(y-x) r(x-z) \bar{\psi}(y) \Gamma_{a} \psi(z),
$$

where we have defined $\Gamma_{a}=\left(\mathbb{1}, i \gamma_{5} \vec{\tau}\right), \tau_{i}$ being the Pauli matrices acting on flavor space. On the other hand, Scheme II $[22,23]$ arises from a separable form of the effective onegluon exchange (OGE) picture. The nonlocal currents $j_{M}^{a}(x)$ are now given by

$$
j_{M}^{a}(x)=\int d^{4} z g(z) \bar{\psi}\left(x+\frac{z}{2}\right) \Gamma_{a} \psi\left(x-\frac{z}{2}\right) .
$$


The functions $r(x-y)$ and $g(z)$ in Eqs. (2) and (3), respectively, are nonlocal covariant form factors characterizing the corresponding interactions. A schematic representation of the four quark interactions in both schemes in momentum space is given in Fig. 1.
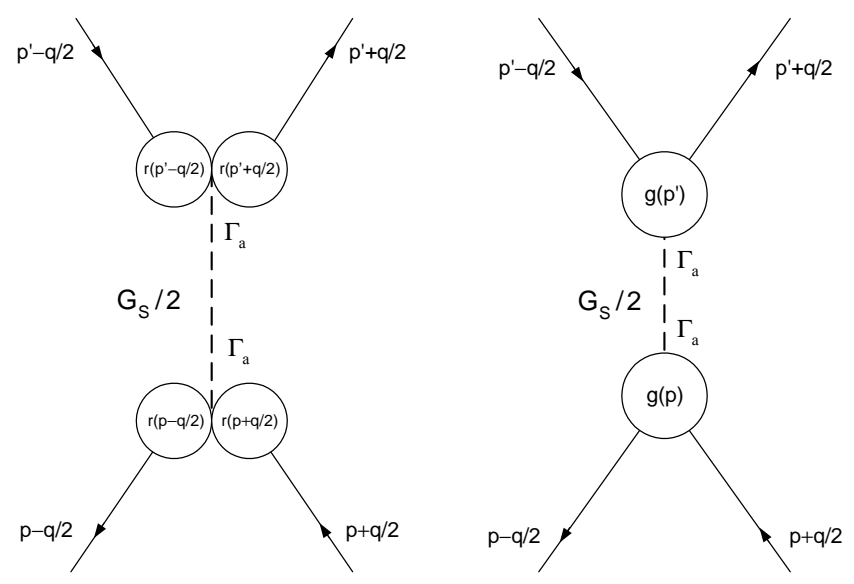

FIG. 1: Schematic representation of the four fermion interaction in Scheme I (left) and Scheme II (right).

In order to deal with meson degrees of freedom, one can perform a standard bosonization of the theory. This is done by considering the corresponding partition function $Z=$ $\int \mathcal{D} \bar{\psi} \mathcal{D} \psi \exp \left[-S_{E}\right]$, and introducing auxiliary fields $S_{a}(x)=$ $(\sigma(x), \vec{\pi}(x))$, where $\sigma(x)$ and $\vec{\pi}(x)$ are scalar and pseudoscalar mesons, respectively. Integrating out the quark fields we get

$$
Z=\int \mathcal{D} \sigma \mathcal{D} \vec{\pi} \exp \left[-S_{E}^{\text {bos }}\right]
$$

where

$$
S_{E}^{\mathrm{bos}}=-\ln \operatorname{det} A+\frac{1}{2 G_{S}} \int \frac{d^{4} p}{(2 \pi)^{4}} S_{a}(p) S_{a}(-p) .
$$

The operator $A$ reads in momentum space [15]

$$
\begin{aligned}
A\left(p, p^{\prime}\right)= & (-p+\bar{m})(2 \pi)^{4} \delta^{(4)}\left(p-p^{\prime}\right) \\
& +h\left(p, p^{\prime}\right) \Gamma_{a} S_{a}\left(p-p^{\prime}\right),
\end{aligned}
$$

where

$$
h\left(p, p^{\prime}\right)= \begin{cases}r\left(p^{2}\right) r\left(p^{\prime 2}\right) & (\text { Scheme I) } \\ g\left[\left(\frac{p+p^{\prime}}{2}\right)^{2}\right] & \text { (Scheme II) } .\end{cases}
$$

We will assume that the $\sigma$ field has a nontrivial translational invariant mean field (MF) value $\bar{\sigma}$, while the MF values of the pseudoscalar fields $\pi_{i}$ are zero. Then we consider fluctuations around these MF values, writing

$$
\begin{aligned}
& \sigma(x)=\bar{\sigma}+\delta \sigma(x) \\
& \vec{\pi}(x)=\delta \vec{\pi}(x)
\end{aligned}
$$

Replacing in the bosonized effective action, and expanding in powers of the meson fluctuations, we get

$$
S_{E}^{\mathrm{bos}}=S_{E}^{\mathrm{MFA}}+S_{E}^{\mathrm{quad}}+\ldots
$$

We will concentrate in the mean field action per unit volume and in the quadratic term for the pseudoscalar fields, $S_{E}^{\text {quad }(\pi)}$. These are given by [15]

$$
\begin{aligned}
\frac{S_{E}^{\mathrm{MFA}}}{V^{(4)}} & =-4 N_{c} \int \frac{d^{4} p}{(2 \pi)^{4}} \ln \left[p^{2}+\Sigma^{2}(p)\right]+\frac{\bar{\sigma}^{2}}{2 G_{S}}, \\
S_{E}^{\mathrm{quad}(\pi)} & =\frac{1}{2} \int \frac{d^{4} p}{(2 \pi)^{4}} G^{-}\left(p^{2}\right) \delta \vec{\pi}(p) \cdot \delta \vec{\pi}(-p) .
\end{aligned}
$$

In these equations we have defined

$$
\begin{aligned}
\Sigma(p) & =\bar{m}+h(p, p) \bar{\sigma} \\
G^{-}\left(p^{2}\right) & =\frac{1}{G_{S}}-8 N_{c} J_{2}\left(p^{2}\right),
\end{aligned}
$$

where $J_{\alpha}\left(p^{2}\right)$ is given by

$$
J_{\alpha}\left(p^{2}\right)=\int \frac{d^{4} q}{(2 \pi)^{4}} \frac{h^{\alpha}\left(q^{+}, q^{-}\right)\left[\left(q^{+} \cdot q^{-}\right)+\Sigma\left(q^{+}\right) \Sigma\left(q^{-}\right)\right]}{\left[\left(q^{+}\right)^{2}+\Sigma^{2}\left(q^{+}\right)\right]\left[\left(q^{-}\right)^{2}+\Sigma^{2}\left(q^{-}\right)\right]}
$$

with $q^{ \pm}=q \pm p / 2$. It is important to point out that, as it is seen from Eq. (13), the nonlocality leads to a momentumdependent selfenergy $\Sigma(p)$.

\section{B. Gap equation and chiral condensates}

In order to find the mean field values $\bar{\sigma}$ one has to minimize the action $S_{E}^{\text {MFA }}$. A straightforward exercise leads to the gap equation

$$
\bar{\sigma}-8 N_{c} G_{S} \int \frac{d^{4} p}{(2 \pi)^{4}} h(p, p) \frac{\Sigma(p)}{p^{2}+\Sigma^{2}(p)}=0 .
$$

The chiral condensates are given by the vacuum expectation values $\langle\bar{q} q\rangle=\langle\bar{u} u\rangle=\langle\bar{d} d\rangle$. They can be easily obtained by performing the variation of $Z^{\mathrm{MFA}}=\exp \left[-S_{E}^{\mathrm{MFA}}\right]$ with respect to the corresponding current quark masses. We obtain

$$
\langle\bar{q} q\rangle=-4 N_{c} \int \frac{d^{4} p}{(2 \pi)^{4}} \frac{\Sigma(p)}{p^{2}+\Sigma^{2}(p)} .
$$

\section{Pion mass and weak decay constant}

According to Eqs. (10) and (12), the pion mass can be obtained by solving the equation

$$
G^{-}\left(-m_{\pi}^{2}\right)=0
$$

From Eq. (12) it is also clear that one has to perform a wave function renormalization of the pseudoscalar fields. The 
renormalized pion field $\overrightarrow{\tilde{\pi}}(p)=\vec{\pi}(p) / Z_{\pi}^{1 / 2}$ is defined by fixing the residue of $G^{-}\left(p^{2}\right)$ at the pion pole, thus one has

$$
Z_{\pi}^{-1}=\left.\frac{d G^{-}(p)}{d p^{2}}\right|_{p^{2}=-m_{\pi}^{2}}
$$

while the $\pi q \bar{q}$ coupling constant $G_{\pi q \bar{q}}$ is given by $Z_{\pi}^{1 / 2}$.

The pion weak decay constant $f_{\pi}$ is given by the matrix element of the axial current $A_{\mu}^{a}(x)$ between the vacuum and the renormalized one-pion state at the pion pole:

$$
\left\langle 0\left|A_{\mu}^{a}(0)\right| \tilde{\pi}^{b}(p)\right\rangle=i \delta^{a b} p_{\mu} f_{\pi} .
$$

In order to obtain an explicitly expression for the axial current, we have to "gauge" the effective action $S_{E}$ by introducing a set of axial gauge fields $\mathcal{A}_{\mu}^{a}(x)$. For a local theory this "gauging" procedure is usually done by performing the replacement

$$
\partial_{\mu} \rightarrow \partial_{\mu}+\frac{i}{2} \gamma_{5} \vec{\tau} \cdot \overrightarrow{\mathcal{A}}_{\mu}(x)
$$

In the present case - owing to the nonlocality of the involved fields - one has to perform an additional replacement, namely

$\gamma_{0} \Gamma_{a} S_{a}(x) \rightarrow\left\{\begin{array}{l}W_{A}(y, x) \gamma_{0} \Gamma_{a} S_{a}(x) W_{A}(x, z) \\ W_{A}(x+z / 2, x) \gamma_{0} \Gamma_{a} S_{a}(x) W_{A}(x, x-z / 2)\end{array}\right.$

where the upper (lower) expression corresponds to Scheme I (II). Here $y$ and $z$ are the integration variables in the definitions of the nonlocal currents [see Eqs. (2) and (3)], and the function $W_{A}(x, w)$ is defined by

$$
W_{A}(x, y)=\mathrm{P} \exp \left[\frac{i}{2} \int_{x}^{y} d s_{\mu} \gamma_{5} \vec{\tau} \cdot \overrightarrow{\mathcal{A}}_{\mu}(s)\right],
$$

where $s$ runs over an arbitrary path connecting $x$ with $y$.

Once the gauged effective action is built, it is easy to get the axial current as the derivative of this action with respect to $\mathcal{A}_{\mu}^{a}(x)$, evaluated at $\overrightarrow{\mathcal{A}}_{\mu}(x)=0$. In this way, after a rather lengthy calculation one obtains [15]

$$
f_{\pi}=Z_{\pi}^{1 / 2} \frac{F\left(-m_{\pi}^{2}\right)-F(0)}{m_{\pi}^{2}}
$$

where $F\left(p^{2}\right)$ is given by

$$
F\left(p^{2}\right)=\bar{m} J_{1}\left(p^{2}\right)+\bar{\sigma} J_{2}\left(p^{2}\right) .
$$

Now, by performing a chiral expansion, it is possible to show [15] that the following relations are satisfied (subindices 0 mean that quantities are evaluated in the chiral limit):

- The Goldberger-Treiman (GT) relation at the quark level: $f_{\pi, 0} G_{\pi q \bar{q}, 0}=\bar{\sigma}_{0}$

- The Gell-Mann-Oakes-Renner (GOR) relation (in the isospin limit): $\bar{m}\langle\bar{u} u+\bar{d} d\rangle_{0}=-f_{\pi, 0}^{2} m_{\pi}^{2}$.

- The chiral relation between $f_{\pi}$ and the anomalous effective coupling $g_{\pi \gamma \gamma}: g_{\pi \gamma, 0}=N_{c} / 12 \pi^{2} f_{\pi, 0}$.

All these relations hold for both Schemes I and II.

\section{MODEL PARAMETERS FOR DEFINITE FORM FACTORS}

In what follows we consider two types of form factors which have been frequently used in the literature, namely the Gaussian (G) form factor

$$
g_{\mathrm{G}}\left(p^{2}\right)=\left[r_{\mathrm{G}}\left(p^{2}\right)\right]^{2}=\exp \left(-p^{2} / \Lambda^{2}\right) .
$$

and the n-Lorenztian $(\mathrm{Ln})$ form factors

$$
g_{\mathrm{Ln}}\left(p^{2}\right)=\left[r_{\operatorname{Ln}}\left(p^{2}\right)\right]^{2}=\frac{1}{1+\left(p^{2} / \Lambda^{2}\right)^{n}} .
$$

Here $n \geq 2$ is an integer that allows us to interpolate between soft form factors (such as the Gaussian one) and sharp form factors as e.g. a step function $g_{\text {Step }}\left(p^{2}\right)=\Theta\left(\Lambda^{2} / p^{2}-1\right)$.

The model parameters $\bar{m}, G_{S}$ and $\Lambda$ are usually determined by fitting the pion mass and decay constant to their empirical values $m_{\pi}=139 \mathrm{MeV}$ and $f_{\pi}=92.4 \mathrm{MeV}$, and fixing the chiral condensate at some phenomenologically acceptable value. Since this last quantity is not well determined, let us consider four representative values within the empirical range, namely $-\langle\bar{q} q\rangle^{1 / 3}=200,220,240$ and $260 \mathrm{MeV}$. With the choice $g\left(p^{2}\right)=\left[r\left(p^{2}\right)\right]^{2}$, both schemes I and II lead to the same expressions for the mean field quantities. However, it should be noticed that (in the case of finite $\bar{m}$ ) for Schemes I and II one gets different expressions for the integrals $J_{\alpha}\left(q^{2}\right)$ that determine the values of $m_{\pi}$ and $f_{\pi}$. Therefore, the fitted model parameters are also expected to be different in both schemes. Our results are shown in Table I, where we quote the parameter values for Schemes I and II, considering different form factors and quark condensates. Interestingly, the current quark mass $\bar{m}$ is basically equal for both schemes, while the remaining two parameters $\left(G_{S} \Lambda^{2}\right.$ and $\left.\Lambda\right)$ might be rather different, especially in the case of soft form factors and low absolute values of the quark condensate. The most striking case corresponds to the L2 form factor: for Scheme II it is not even possible to find a parameter set leading to a condensate of either $-\langle\bar{q} q\rangle^{1 / 3}=200 \mathrm{MeV}$ or $220 \mathrm{MeV}$. For the Gaussian form factor, although we do find compatible parameter sets, the obtained values of $\Lambda$ in Scheme II for the case of low condensates are too low to be reliable for phenomenological applications.

An important feature of nonlocal chiral quark models is that the analytic structure of the quark propagator $S(p)$ in the complex plane is richer than in the local NJL theory. Due to the momentum dependence of the selfenergies, there might be a rather complicate structure of poles and cuts [10, 11, 21]. For $\vec{p}=0$, the positions of the poles are given by the solutions of

$$
p_{4}^{2}+\left[\bar{m}+\bar{\sigma} g^{2}\left(p_{4}^{2}\right)\right]^{2}=0 .
$$

In Table II we quote the values obtained for $\bar{\sigma}$ and the position of the first pole of the quark propagator, for both Schemes I and II, and different form factors and quark condensates. As discussed in the literature [21, 24], the absence of purely imaginary poles in the quark propagator can be understood 
TABLE I: Model parameters for various form factors leading to some acceptable values of the chiral condensate ( $\bar{m}$ and $\Lambda$ given in $\mathrm{MeV}$ )

\begin{tabular}{|c|c|c|c|c|c|c|c|}
\hline \multirow[t]{2}{*}{$-\langle q \bar{q}\rangle^{1 / 3}$} & \multirow[t]{2}{*}{ Form factor } & \multicolumn{3}{|c|}{ Scheme I } & \multicolumn{3}{|c|}{ Scheme II } \\
\hline & & $\bar{m}$ & $G_{S} \Lambda^{2}$ & $\Lambda$ & $\bar{m}$ & $G_{S} \Lambda^{2}$ & $\Lambda$ \\
\hline \multirow[t]{4}{*}{200} & G & 9.7 & 18.82 & 651.9 & 9.8 & 71.11 & 459.7 \\
\hline & $\mathrm{L} 2$ & 9.7 & 12.45 & 539.9 & - & - & - \\
\hline & L5 & 9.6 & 8.71 & 799.0 & 9.6 & 13.38 & 660.2 \\
\hline & L10 & 9.8 & 7.57 & 885.0 & 9.7 & 10.68 & 716.5 \\
\hline \multirow[t]{4}{*}{220} & $\bar{G}$ & 7.4 & 16.98 & 772.0 & 7.4 & 29.06 & 604.0 \\
\hline & L2 & 7.4 & 10.98 & 642.2 & - & - & - \\
\hline & L5 & 7.4 & 8.40 & 924.4 & 7.4 & 10.34 & 790.3 \\
\hline & L10 & 7.5 & 7.47 & 1019.3 & 7.4 & 9.06 & 843.2 \\
\hline \multirow[t]{4}{*}{240} & G & 5.8 & 15.82 & 902.4 & 5.8 & 20.65 & 752.2 \\
\hline & L2 & 5.8 & 10.14 & 751.8 & 5.8 & 16.06 & 586.8 \\
\hline & L5 & 5.8 & 8.20 & 1059.2 & 5.8 & 9.27 & 925.7 \\
\hline & L10 & 5.8 & 7.39 & 1163.0 & 5.8 & 8.36 & 978.6 \\
\hline \multirow[t]{4}{*}{260} & G & 4.6 & 15.08 & 1042.2 & 4.6 & 17.53 & 903.4 \\
\hline & L2 & 4.6 & 9.61 & 868.0 & 4.6 & 11.77 & 736.1 \\
\hline & L5 & 4.6 & 8.07 & 1202.8 & 4.6 & 8.73 & 1067.7 \\
\hline & $\overline{\mathrm{L} 10}$ & 4.6 & 7.34 & 1315.6 & 4.6 & 7.98 & 1122.5 \\
\hline
\end{tabular}

as a realization of quark confinement. In fact, previous analysis show that for most applications only the first pole, i.e. the closest one to the real axis, is phenomenologically relevant $[10,11,21]$. In the case of Scheme I, as one can see from Table II, for the cases considered the first pole is complex only for a condensate of $200 \mathrm{MeV}$ and form factors $\mathrm{G}$ and L10 (we denote by "complex" a pole which has both nonvanishing real and imaginary components). Moreover, the case of L10 is not phenomenologically viable in view of the low value of $\bar{\sigma}$. Thus, we conclude that if one wants to avoid (following confinement arguments) the presence of low lying purely imaginary poles, the only acceptable situation for Scheme I is that which corresponds to a soft (Gaussian) form factor and a low value of $-\langle\bar{q} q\rangle^{1 / 3}$. Sharper form factors would be excluded, leading in all cases to too small values of $\bar{\sigma}$. In the case of Scheme II, one observes that sharp form factors also tend to yield small values of $\bar{\sigma}$, the sole exceptions being the cases L5 and L10 with $-\langle\bar{q} q\rangle^{1 / 3}=200 \mathrm{MeV}$. In these cases, however, the situation is also problematic, since the first poles, although complex, have a very small imaginary part. This implies the existence of a very low $\bar{q} q$ pseudo-threshold, which would require additional prescriptions to preserve meson stability already at energies of a few hundreds of $\mathrm{MeV}$ [11]. Given the above discussed limitations of Scheme II for soft form factors and low absolute values of the condensate, we conclude that the sets corresponding to G and L2 form factors and values of $-\langle\bar{q} q\rangle^{1 / 3}$ between 240 and $260 \mathrm{MeV}$ would provide the more acceptable results.

\section{PHASE TRANSITIONS IN THE $T-\mu$ PLANE}

After the description of the main features of nonlocal chiral quark models presented in the previous sections, we turn now to analyze the thermodynamical properties of these theo-
TABLE II: Values for $\bar{\sigma}$ and location of the first pole of the quark propagator for various models (quantities given in $\mathrm{MeV}$ )

\begin{tabular}{|c|c|c|c|c|c|}
\hline \multirow[t]{2}{*}{$-\langle q \bar{q}\rangle^{1 / 3}$} & \multirow[t]{2}{*}{ Form factor } & \multicolumn{2}{|c|}{ Scheme I } & \multicolumn{2}{|c|}{ Scheme II } \\
\hline & & $\bar{\sigma}$ & First pole & $\bar{\sigma}$ & First pole \\
\hline \multirow[t]{4}{*}{200} & G & 318 & $180+454 i$ & 1356 & $499+241 i$ \\
\hline & $\mathrm{L} 2$ & 296 & $284 i$ & - & - \\
\hline & L5 & 181 & $191 \mathrm{i}$ & 366 & $640+169 i$ \\
\hline & L10 & 149 & $875+131 i$ & 302 & $711+98 i$ \\
\hline \multirow{4}{*}{220} & $\mathrm{G}$ & 282 & $356 \mathrm{i}$ & 620 & $421+372 i$ \\
\hline & $\mathrm{L} 2$ & 259 & $259 i$ & - & - \\
\hline & L5 & 173 & $180 \mathrm{i}$ & 276 & $757+217 i$ \\
\hline & L10 & 145 & $152 \mathrm{i}$ & 248 & $835+120 i$ \\
\hline \multirow[t]{4}{*}{240} & $\mathrm{G}$ & 255 & $288 \mathrm{i}$ & 424 & $288+510 i$ \\
\hline & L2 & 233 & $237 i$ & 475 & $485+304 i$ \\
\hline & L5 & 166 & $153 i$ & 238 & $243 \mathrm{i}$ \\
\hline & L10 & 142 & $147 i$ & 221 & $968+142 i$ \\
\hline \multirow[t]{4}{*}{260} & G & 235 & $255 i$ & 339 & $430 i$ \\
\hline & L2 & 216 & $219 i$ & 330 & $323 \mathrm{i}$ \\
\hline & L5 & 160 & $172 \mathrm{i}$ & 215 & $220 i$ \\
\hline & L10 & 139 & $143 \mathrm{i}$ & 204 & $1110+165 i$ \\
\hline
\end{tabular}

ries, studying the different phases that can be expected at finite temperature $T$ and quark chemical potential $\mu=\mu_{B} / 3$. First we will present the results obtained in the case of isospin symmetric quark matter, and then we will analyze how the phase diagram gets modified under the conditions of color and electric charge neutrality and $\beta$ equilibrium.

\section{A. Isospin symmetric quark matter}

We begin by considering an Euclidean action as that given in Eq. (1), with the addition of a nonlocal quark-quark interaction

$$
\mathcal{L}_{q q}=-\frac{H}{2}\left[j_{D}^{a}(x)\right]^{\dagger} j_{D}^{a}(x) .
$$

Here the diquark currents are given by

$j_{D}^{a}(x)=\left\{\begin{array}{l}\int d^{4} y d^{4} z r(y-x) r(x-z) \bar{\psi}_{C}(y) i \gamma_{5} \tau_{2} \lambda_{a} \psi(z) \\ \int d^{4} z g(z) \bar{\psi}\left(x+\frac{z}{2}\right) i \gamma_{5} \tau_{2} \lambda_{a} \psi\left(x-\frac{z}{2}\right)\end{array}\right.$,

the upper (lower) expression corresponding to Scheme I (Scheme II). We have defined $\psi_{C}(x)=\gamma_{2} \gamma_{4} \bar{\psi}^{T}(x)$, while $\lambda_{a}$, with $a=2,5,7$, are Gell-Mann matrices acting on color space.

The effective interaction in Eq. (28) might arise via Fierz rearrangement from some underlying more fundamental interactions, and is understood to be used - at the mean field level - in the Hartree approximation. In general, the ratio of coupling constants $H / G_{S}$ would be determined by these microscopic couplings; for example, OGE interactions, as well as instanton model interactions, lead to $H / G_{S}=0.75$. Since the precise derivation of the effective couplings from QCD is not known, there is a significant theoretical uncertainty in this value. We will consider $H / G_{S}=0.75$ as a reference value, 
and then we will analyze the sensitivity of our results when the ratio varies within a range from 0.5 to 1 .

The partition function of the system at temperature $T$ and quark chemical potentials $\mu$ is given by

$$
Z=\int D \bar{\psi} D \psi e^{-S_{E}(\mu, T)}
$$

where $S_{E}(\mu, T)$ is obtained from the Euclidean action at zero $T$ and $\mu$ just by performing a Fourier transformation into momentum space, and then making the replacements

$$
p_{4} \rightarrow \omega_{n}-i \mu, \quad \int \frac{d^{4} p}{(2 \pi)^{4}} \rightarrow T \sum_{n=-\infty}^{\infty} \int \frac{d^{3} \vec{p}}{(2 \pi)^{3}}
$$

Here $\omega_{n}$ are the Matsubara frequencies corresponding to fermionic modes, $\omega_{n}=(2 n+1) \pi T$. We are implicitly assuming that quark interactions depend on $T$ and $\mu$ only through the arguments of the form factors, while $G_{S}$ and $H$ remain unchanged.

As in the previous sections, we proceed by performing a standard bosonization of the theory. In addition to the bosonic fields $\sigma$ and $\pi_{a}$ now we have to introduce diquark fields $\Delta_{a}$, with MF values $\bar{\Delta}_{a}$. Owing to color symmetry, one can choose an arbitrary orientation for the nonzero MF value of the diquark fields in color space. Thus we adopt the usual ansatz in which one has $\bar{\Delta}_{5}=\bar{\Delta}_{7}=0, \bar{\Delta}_{2}=\bar{\Delta}$. This leaves a residual symmetry under a color SU(2) subgroup. Then, making use of the Nambu-Gorkov formalism, the mean field thermodynamical potential per unit volume can be written as [13]

$$
\begin{aligned}
\Omega^{\mathrm{MFA}} & =-\frac{T}{V} \ln Z^{\mathrm{MFA}}= \\
& =\frac{\bar{\sigma}^{2}}{2 G_{S}}+\frac{|\bar{\Delta}|^{2}}{2 H}-\frac{T}{2} \sum_{n=-\infty}^{\infty} \int \frac{d^{3} \vec{p}}{(2 \pi)^{3}} \ln \operatorname{det}\left[\frac{S^{-1}}{T}\right]
\end{aligned}
$$

where the inverse propagator $S^{-1}(\bar{\sigma}, \bar{\Delta})$ is a $48 \times 48$ matrix in Dirac, flavor, color and Nambu-Gorkov spaces. This propagator can be written in the compact form

$$
S^{-1}=\left(\begin{array}{cc}
-p^{+}+\Sigma\left(p^{+}\right) & i \gamma_{5} \tau_{2} \lambda_{2} \Delta\left(p^{+}, p^{-}\right) \\
i \gamma_{5} \tau_{2} \lambda_{2} \Delta\left(p^{-}, p^{+}\right) & -p^{-}+\Sigma\left(p^{-}\right)
\end{array}\right)
$$

where we have used the definitions

$$
\begin{aligned}
p^{ \pm \mu} & =\left(\omega_{n} \mp i \mu, \vec{p}\right), \\
\Delta\left(p^{ \pm}, p^{\mp}\right) & =\bar{\Delta} h\left(p^{ \pm}, p^{\mp}\right) .
\end{aligned}
$$

For finite values of the current quark mass, $\Omega^{\mathrm{MFA}}$ turns out to be divergent. We regularize it by defining

$$
\Omega_{(\mathrm{reg})}^{\mathrm{MFA}}=\Omega^{\mathrm{MFA}}-\Omega^{\text {free }}+\Omega_{(\mathrm{reg})}^{\mathrm{free}},
$$

where $\Omega^{\text {free }}$ is obtained from Eq. (31) by setting $\bar{\Delta}=\bar{\sigma}=0$, and $\Omega_{(\text {reg })}^{\text {free }}$ is the usual regularized form for the thermodynamical potential of a free fermion gas. Now the mean field values $\bar{\sigma}$ and $\bar{\Delta}$ can be obtained from the coupled gap equations

$$
\frac{d \Omega_{(\mathrm{reg})}^{\mathrm{MFA}}}{d \bar{\Delta}}=0, \quad \frac{d \Omega_{(\mathrm{reg})}^{\mathrm{MFA}}}{d \bar{\sigma}}=0 .
$$

Let us analyze the features of the phase diagrams and the behavior of relevant physical quantities for Schemes I and II, taking a definite form factor. According to the discussion in Sect. III, we will choose Gaussian form factors $g_{G}\left(p^{2}\right)$, and a rather high value of the condensate, namely $\langle 0|\bar{q} q| 0\rangle^{1 / 3}=$ $-250 \mathrm{MeV}$. With this choice, following the procedure described in Sect. III, the model parameters are found to be $m=$ $5.14 \mathrm{MeV}, \Lambda=971 \mathrm{MeV}$ and $G_{S} \Lambda^{2}=15.41$ for Scheme I and $m=5.12 \mathrm{MeV}, \Lambda=827 \mathrm{MeV}$ and $G_{S} \Lambda^{2}=18.78$ for Scheme II. As stated above, we will take for now $H / G_{S}=0.75$. The models defined by these parameter sets will be called from now on Model I and Model II.
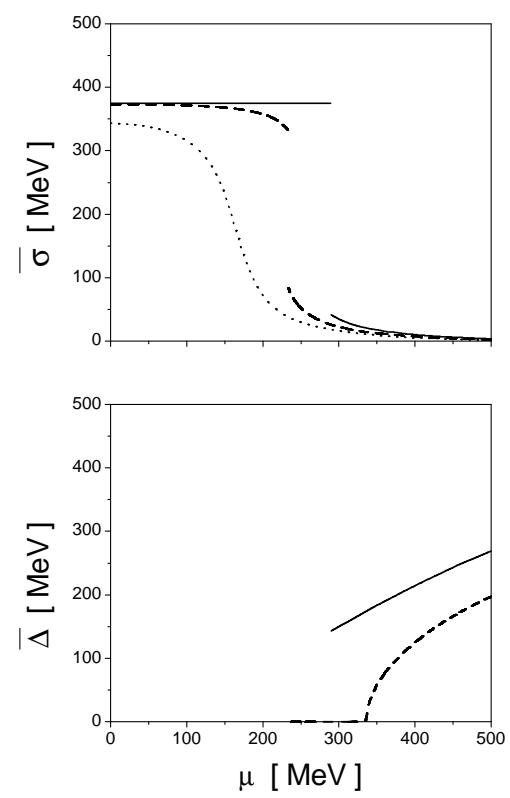

FIG. 2: Behavior of the mean fields $\bar{\sigma}$ and $\bar{\Delta}$ as functions of the chemical potential, for three different values of the temperature. Full lines correspond to $T=0$, dashed lines to $T=70 \mathrm{MeV}$ and dotted lines to $T=100 \mathrm{MeV}$. The data correspond to Model II.

Our results for $\bar{\sigma}$ and $\bar{\Delta}$ are shown in Fig. 2, where we plot these quantities as functions of $\mu$ for different representative values of the temperature. For definiteness we have chosen Model II, which (as discussed above) seems to be phenomenologically preferred. For $T=0$ (solid lines), at low chemical potentials the system is in a chiral symmetry broken phase (CSB), where quarks acquire large dynamical masses and $\bar{\Delta}=0$. By increasing the chemical potential one reaches a first order phase transition at a critical value $\mu_{c}=290 \mathrm{MeV}$. At this point the chiral symmetry is approximately restored, and one observes a finite gap on the MF value $\bar{\Delta}$. Thus the system gets into a so-called two flavor superconducting (2SC) phase. The nonzero value $\bar{\Delta}$ breaks color symmetry, causing quarks of two colors -say read and green- to get paired in a similar way as cooper pairs are formed in superconducting materials. The remaining -blue- quarks are kept unpaired, as a consequence of the residual SU(2) color symmetry. It is found that the order of magnitude of this diquark gap is around one to 
two hundred MeV for both Models I and II.

When the temperature is increased one has a similar situation, until a "triple point" temperature $T_{3 P}$ is reached. For temperatures slightly higher than this triple point $\left(T_{3 P}=33\right.$ MeV for Model I, $56 \mathrm{MeV}$ for Model II), the chiral and superconducting transitions do not occur simultaneously, thus one can define two critical values of the chemical potential, $\mu_{c 1}$ and $\mu_{c 2}$. At $\mu_{c 1}$ one finds a first order chiral phase transition, entering a normal quark matter (NQM) phase, and then at $\mu_{c 2}$ one reaches a second order transition into the $2 \mathrm{SC}$ phase. In Model II this is the situation e.g. for $T=70 \mathrm{MeV}$, which corresponds to the dashed curves in Fig. 2. Here $\mu_{c 1}=233$ $\mathrm{MeV}$ and $\mu_{c 2}=335 \mathrm{MeV}$. Finally, if the temperature is further increased, one arrives at an "end point" (EP) where the first order transition from CSB to NQM phases becomes a smooth crossover. For Models I and II this temperature is located at $34 \mathrm{MeV}$ (almost coincident with $T_{3 P}$ ) and $81 \mathrm{MeV}$ respectively. The dotted curve in Fig. 2, corresponding to Model II and $T=100 \mathrm{MeV}$, illustrates this situation. No 2SC transition is observed up to $\mu=500 \mathrm{MeV}$ in this case.

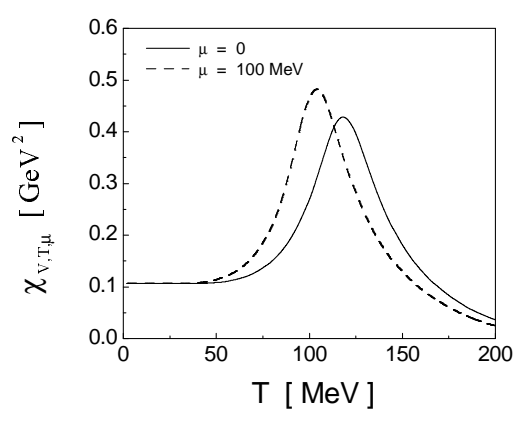

FIG. 3: Typical behavior of the susceptibility $\chi_{v, T, \mu}$, for two fixed values of the chemical potential. Curves correspond to Model I.

In order to properly define the transition temperatures for these smooth crossovers, we have taken into account the behavior of the chiral susceptibility $\chi_{V, T, \mu}$, defined as

$$
\chi_{V, T, \mu}=-\left(\frac{\partial\langle\bar{q} q\rangle(T, \mu, \bar{m})}{\partial \bar{m}}\right)_{T, \mu} .
$$

For a fixed value of $\mu$, the phase transition temperature can be defined as the point where the susceptibility shows a peak, the sharpness of this peak serving as measure of the steepness of the crossover [12]. An illustration of this behavior is given in Fig. 3, where it is seen how the critical temperature decreases with increasing $\mu$. Qualitatively, the situation is similar for both Models I and II. In both cases it is observed that the critical temperature $T_{c}(\mu=0)$ lies between 120 to $140 \mathrm{MeV}$. This is a value quite low in comparison with lattice QCD calculations, which lead to values between 160 and $200 \mathrm{MeV}$ [25].

The described features of the phase diagrams for Models I and II can be visualized in the graphs shown in Fig. 4, where we plot the transition curves on the $T-\mu$ plane, showing the regions corresponding to the different phases and the position of triple and end points. In the graphs, solid and dotted lines correspond to the mentioned first order and crossover transi- tions, respectively, while dashed lines correspond to the second order phase transition between NQM and $2 \mathrm{SC}$ regions.
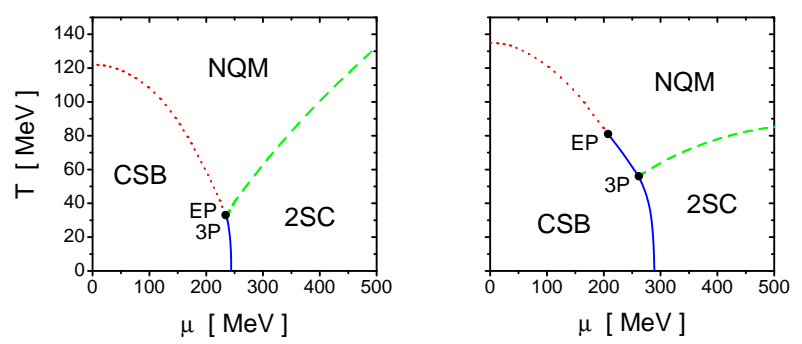

FIG. 4: Phase diagrams for symmetric matter corresponding to Models I (left) and II (right), with $H / G=0.75$. Full and dashed lines indicate first and second order phase transition curves respectively, while dotted lines correspond to the crossover transitions. Different phases are denoted as NQM (normal quark matter phase), CSB (chiral symmetry broken phase) and 2SC (two-flavor superconducting phase). $\mathrm{EP}$ and $3 \mathrm{P}$ denote the end points and triple points respectively.

The dependence of these results on the value of the ratio $H / G_{S}$ has been also analyzed [13]. On one hand, it is seen that in general the 2SC phase region becomes larger when the ratio $H / G_{S}$ is increased. This is not surprising, since $H$ is the effective coupling governing the quark-quark interaction that gives rise to the quark pairing. As a general conclusion, it can be stated that, provided the ratio $H / G$ is not too low, the nonlocal schemes favor the existence of color superconducting phases at low temperatures and moderate chemical potentials. This is in contrast e.g. with the situation in the NJL model [26], where the existence of a $2 \mathrm{SC}$ phase turns out to be rather dependent on the input parameters. In addition, our results are qualitatively different from those obtained in the case of noncovariant nonlocal models [27]: in those theories, above the chiral phase transition the NQM phase is favored for intermediate values of $H$, and the 2SC quark matter phase can be found only for $H / G_{S} \approx 1$. In contrast, in our models the NQM phase can be favored at low temperatures only for $H / G_{S} \lesssim 0.5$.

Finally, we have studied the dependence of the phase transitions on the model parameters, changing the input value of the chiral condensate within the phenomenologically acceptable range $220 \mathrm{MeV} \leq-\langle 0|\bar{q} q| 0\rangle^{1 / 3} \leq 260 \mathrm{MeV}$. From this analysis, it is found that the qualitative features of the phase diagrams are not significantly modified. In particular, it is seen that one finds in general color superconducting phases at low temperatures and moderate chemical potentials for intermediate values of the ratio $H / G_{S}$. In addition, the values for the critical temperature at $\mu=0$ are quite stable, yielding about $120 \mathrm{MeV}$ for Model I and $140 \mathrm{MeV}$ for Model II. Considering the values obtained from lattice calculations this favors in principle the description given by Model II, though the critical temperature is still relatively low. 


\section{B. Quark matter under compact star conditions}

As stated in the Introduction, the analysis of the phase diagram for strongly interacting matter has important applications in astrophysics. In particular, the region of low temperatures and moderate baryon chemical potentials is very interesting for the description of compact star cores. Thus, in the framework of nonlocal chiral quark models, it is worth to analyze the features of the phase diagram under compact star conditions, i.e., under the requirements of color and electric charge neutrality together with $\beta$ equilibrium.

Color neutrality arises basically from the interaction between matter and gauge interactions. In our effective quark models the effect of these interactions is just taken into account through the quark-antiquark and quark-quark couplings, while the model does not include gauge field dynamics. However, it is possible to account for the effect of gauge interactions leading to color neutrality by the introduction of effective color chemical potentials. In addition, the conditions of charge neutrality and $\beta$ equilibrium require the inclusion of electrons and the introduction of chemical potentials associated with quark electric charges. Thus, the mean field thermodynamical potential for quark matter can be written as a generalization of Eq. (31) [14], in which for each quark of flavor $f$ and color $c$ one considers a different "momentum"

$$
p^{ \pm \mu}=\left(\omega_{n} \mp \mu_{f c}, \vec{p}\right)
$$

If the system is in chemical equilibrium, it can be seen that the effective quark chemical potentials $\mu_{f c}$ are in general not independent. Indeed, within the previously introduced ansatz for the mean field values $\bar{\Delta}_{a}$, only one color chemical potential is needed to ensure color charge neutrality, and all $\mu_{f c}$ can be written in terms of only three independent quantities: the baryonic chemical potential $\mu_{B}$, a quark electric chemical potential $\mu_{Q_{q}}$ and a color chemical potential $\mu_{8}$. Defining as before $\mu=\mu_{B} / 3$, the corresponding relations read [14]

$$
\begin{aligned}
\mu_{u r}=\mu_{u g} & =\mu+\frac{2}{3} \mu_{Q_{q}}+\frac{1}{3} \mu_{8} \\
\mu_{d r}=\mu_{d g} & =\mu-\frac{1}{3} \mu_{Q_{q}}+\frac{1}{3} \mu_{8} \\
\mu_{u b} & =\mu+\frac{2}{3} \mu_{Q_{q}}-\frac{2}{3} \mu_{8} \\
\mu_{d b} & =\mu-\frac{1}{3} \mu_{Q_{q}}-\frac{2}{3} \mu_{8} .
\end{aligned}
$$

Notice that, as in the isospin symmetric case, red and green quarks remain indistinguishable owing to the residual SU(2) color symmetry.

We also have to take into account the presence of electrons and the condition of $\beta$ equilibrium. The electrons can be easily included as a gas of free Dirac particles, leading to a full mean field grand canonical thermodynamical potential

$$
\Omega^{\text {full }}=\Omega_{(\mathrm{reg})}^{\mathrm{MFA}}+\Omega^{e},
$$

where

$$
\Omega^{e}=-\frac{1}{12 \pi^{2}}\left(\mu_{e}^{4}+2 \pi^{2} T^{2} \mu_{e}^{2}+\frac{7 \pi^{4}}{15} T^{4}\right)
$$

$\mu_{e}$ being the electron chemical potential (for simplicity we have neglected here the electron mass). Finally, the condition of $\beta$ equilibrium arises from the $\beta$ decay reaction $d \rightarrow$ $u+e+\bar{v}_{e}$. Then, assuming that antineutrinos escape from the stellar core, quark and electron chemical potentials appear to be related by

$$
\mu_{d c}-\mu_{u c}=-\mu_{Q_{q}}=\mu_{e} .
$$

Under this condition all effective fermion chemical potentials can be written in terms of $\mu, \mu_{8}$ and $\mu_{e}$.

Now the conditions of electric and color charge neutrality are imposed by requiring that the electric charge density $\rho_{Q_{t o t}}$ and the diagonal color charge densities $\rho_{3}$ and $\rho_{8}$ vanish simultaneously [28]. The condition $\rho_{3}=0$ is trivially satisfied, while for the charge densities $\rho_{Q_{t o t}}$ and $\rho_{8}$ one has

$$
\begin{aligned}
\rho_{Q_{t o t}} & =\rho_{Q_{q}}-\rho_{e}=\sum_{c=r, g, b}\left(\frac{2}{3} \rho_{u c}-\frac{1}{3} \rho_{d c}\right)-\rho_{e}=0, \\
\rho_{8} & =\frac{1}{\sqrt{3}} \sum_{f=u, d}\left(\rho_{f r}+\rho_{f g}-2 \rho_{f b}\right)=0,
\end{aligned}
$$

where

$$
\begin{gathered}
\rho_{e}=-\frac{\partial \Omega^{\text {full }}}{\partial \mu_{e}}=-\frac{\partial \Omega^{e}}{\partial \mu_{e}}, \\
\rho_{f c}=-\frac{\partial \Omega^{\text {full }}}{\partial \mu_{f c}}=-\frac{\partial \Omega_{(\mathrm{reg})}^{\mathrm{MFA}}}{\partial \mu_{f c}} .
\end{gathered}
$$

Consequently, in the physical situation we are interested in, for each value of $T$ and $\mu$ we should find the values of $\bar{\Delta}$, $\bar{\sigma}, \mu_{e}$ and $\mu_{8}$ that solve the generalized gap equations (36), supplemented by Eqs. (42) and (43).

To present definite numerical results we consider once again the Models I and II presented in the previous subsection. Now, in addition to the MF values $\bar{\sigma}$ and $\bar{\Delta}$, it is important to analyze the behavior of the effective chemical potentials $\mu_{e}$ and $\mu_{8}$. The results are shown in Fig. 5, where we plot these quantities as functions of $\mu$ for different representative values of the temperature. The left (right) panel in the figure corresponds to Model I (II), and solid, dashed and dotted lines correspond to $T=0,40 \mathrm{MeV}$ and $100 \mathrm{MeV}$ respectively. The corresponding phase diagrams are shown in Fig. 6.

It can be seen that the phase transitions are in many aspects qualitatively similar as those presented in the previous section for the case of symmetric quark matter. However, one has now some important new features. For low temperatures and large chemical potentials (the region of interest for compact star applications), it is seen that the system gets into a phase which is not homogeneous but favors the coexistence of NQM and 2SC phases. In this mixed phase zone the electric neutrality is realized globally: the different phases have opposite electric charges which cancel each other, with a common equilibrium pressure. The chemical potential $\mu_{e}$, which for $T=0$ vanishes in the CSB phase but is nonzero in the mixed phase, shows a discontinuity across the first order transition. The same happens with the color chemical potential $\mu_{8}$ in the 2SC component of the mixed phase (lines marked with 

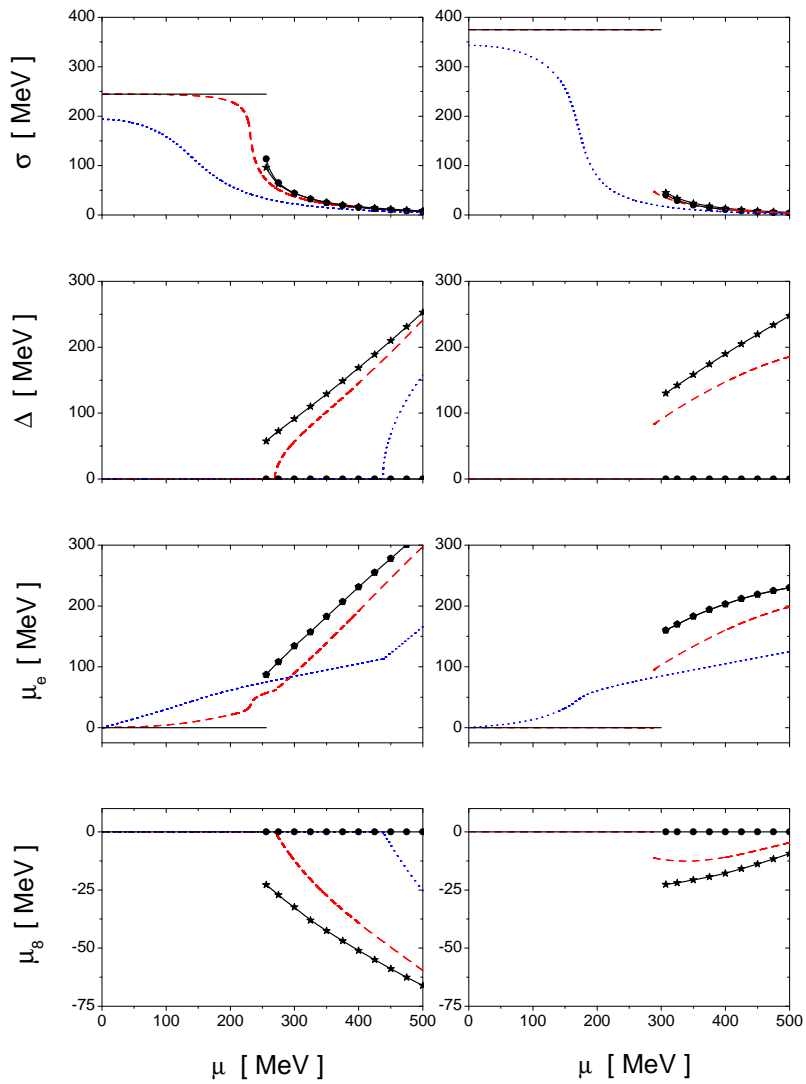

FIG. 5: Behavior of $\bar{\sigma}, \bar{\Delta}, \mu_{e}$ and $\mu_{8}$ for Models I (left) and II (right) as functions of the chemical potential, for three different values of the temperature. Solid lines correspond to $T=0$, dashed lines to $T=40 \mathrm{MeV}$ and dotted lines to $T=100 \mathrm{MeV}$. In the case of $T=0$, lines marked with stars and dots correspond to 2SC and NQM phases respectively.

stars in the figure). As expected, the growth of $\left|\mu_{8}\right|$ in this phase is approximately proportional to that of $\bar{\Delta}$, which governs the amount of breakdown of the color symmetry due to quark pairing. Then, when the temperature is increased (see e.g. dashed curves in Fig. 5, corresponding to $T=40 \mathrm{MeV}$ ), this mixed phase is no longer favored and the system goes into a pure $2 \mathrm{SC}$ phase. For $T=40 \mathrm{MeV}$, this shows up as a second order transition in the case of Model I, and a first order transition in the case of Model II. Now, for both models, when one moves along the first order transition line from $T=0$ towards higher temperatures, one arrives at a triple point and then at an end point, as in the case of symmetric matter.

Another new feature is the presence of the so-called gapless 2SC (g2SC) phases. As it is shown in Fig. 6, g2SC phases are favored in a narrow band close to the $2 \mathrm{SC}-\mathrm{NQM}$ phase border. In this region, in addition to the two gapless modes corresponding to the unpaired blue quarks, the presence of flavor asymmetric chemical potentials $\mu_{d c}-\mu_{u c} \neq 0$ gives rise to another two gapless fermionic quasiparticles [29]. Although the corresponding dispersion relations cannot be derived analytically owing to the nonlocality of the interactions, the border of the g2SC region can be found numerically. This is done by
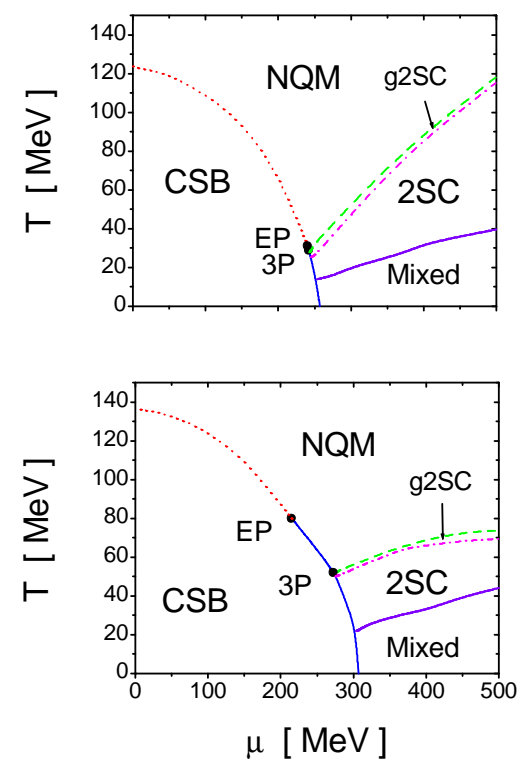

FIG. 6: Phase diagrams for Models I (up) and II (down). Solid and dashed lines indicate first and second order phase transition curves respectively, dotted lines correspond to crossover-like transitions, and dashed lines delimit the gapless 2SC band. Different phases are denoted as NQM (normal quark matter phase), CSB (chiral symmetry broken phase) and 2SC (two-flavor superconducting phase), while the regions marked as "Mixed" correspond to the NQM-2SC mixed phase. EP and 3P denote the end points and triple points respectively.

determining whether for some value of $|\vec{p}|$ the imaginary part of some of the poles of the Euclidean quark propagator vanish in the complex $p_{4}$ plane. In the case of Models I and II the size of the g2SC band is too small to lead to sizeable effects. However, the region appears to be considerable enlarged if we consider models with a lower value of $H / G_{S}$ (see Ref. [14]). In any case for these kind of models it is seen that the g2SC phase never extends up to zero temperatures, thus it should not represent a robust feature for compact star applications.

After having analyzed the quark matter phase diagrams, it is natural to ask whether such a state of matter can exist in the interior of cold compact stars. Unfortunately, a consistent relativistic approach to the quark-hadron phase transition, where hadrons appear as bound states of quarks, has not been developed up to now. To address this problem, in Ref. [16] we have applied a so-called two-phase description, in which the nuclear matter phase is treated within the relativistic Dirac-Brueckner-Hartree-Fock (DBHF) approach (see e.g. Ref. [30]) and the transition to a quark matter phase is obtained by a Maxwell construction.

From the phase diagrams in Fig. 6 it is found that the models predict a relatively low critical density $\mu_{c}$ at $T=0$, hence some extra repulsion would be welcome in order to obtain a more realistic value. We have found that this can be achieved by including some small additional interaction in the omega vector meson channel, which does not affect other qualitative features of the phase diagrams [16]. For consistency, this new 
vector interaction has been included as a nonlocal four point coupling, driven by a new coupling constant $G_{V}$. Within these approaches for the nuclear and quark matter descriptions we have obtained the corresponding equations of state and determined the mass and structure of stars by solving the TolmanOppenheimer-Volkov equations (we have restricted ourselves to spherical, nonrotating objects). Our results are found to be in agreement with modern observational constraints, including compact star masses above $2 M_{\odot}$, if one takes values of $H / G_{S}$ close to 0.75 and values of $G_{V} / G_{S} \sim 0.05$. In addition, the corresponding isospin symmetric equations of state are shown to be consistent with flow data analyses of heavy ion collisions. Therefore, as a main conclusion, it can be said that compact stars with quark matter cores are not ruled out by observations.

\section{DESCRIPTION OF CONFINEMENT: COUPLING WITH THE POLYAKOV LOOP}

One of the weakest aspects of the NJL model is that it does not offer an adequate description of the confinement/deconfinement transitions. This is shared by the effective nonlocal chiral quark models considered here. In addition, as shown in the previous section, in our nonlocal models the critical temperatures for the chiral phase transition $T_{C}(\mu=0)$ are typically below the values obtained within lattice QCD. We show that both problems can be addressed by considering the coupling of quarks with the Polyakov loop. This represents a sort of extension of the so-called PNJL model, now including nonlocal four point interactions. The traced Polyakov loop can be taken as an order parameter for the confinement/deconfinement transition.

Let us consider the Euclidean action (1) for the chiral nonlocal models, extended to finite $T$ and keeping $\mu=0$. The coupling of fermions to the Polyakov loop can be implemented through a covariant derivative $D_{\mu} \equiv \partial_{\mu}-i A_{\mu}$ in the fermion kinetic term. We will assume that the quarks move in a constant background color gauge field $A_{4}=i A_{0}=i g \delta_{\mu 0} G_{a}^{\mu} \lambda^{a} / 2$, where $G_{a}^{\mu}$ are the SU(3) color gauge fields. Then the traced Polyakov loop is given by $\Phi=\frac{1}{3} \operatorname{Tr} \exp (i \beta \phi)$, with $\beta=1 / T$. We will work in the so-called Polyakov gauge, in which the matrix $\phi$ is given a diagonal representation $\phi=\phi_{3} \lambda_{3}+\phi_{8} \lambda_{8}$, which leaves only two independent variables, $\phi_{3}$ and $\phi_{8}$. Finally, the action also includes an effective potential $\mathcal{U}(\Phi, T)$ that accounts for gauge field self-interactions.

Once again we will consider the thermodynamical potential at the mean field level. Owing to the charge conjugation properties of the QCD Lagrangian [31], the MF value of the Polyakov loop field $\Phi$ is expected to be a real quantity. Assuming that $\phi^{3}$ and $\phi^{8}$ are real-valued fields [32], this implies $\bar{\phi}^{8}=0, \bar{\Phi}=\left[2 \cos \left(\bar{\phi}^{3} / T\right)+1\right] / 3$. On the other hand, the function $\mathcal{U}(\bar{\Phi}, T)$ can be fitted by taking into account group theory constraints together with lattice results, from which one can estimate the temperature dependence. In our calculations we have taken for $\mathcal{U}(\bar{\Phi}, T)$ the form given in Ref. [32].

The values of $\bar{\phi}_{3}$ and $\bar{\sigma}$ can be now obtained as usual by the minimization of the regularized MF thermodynamical poten-
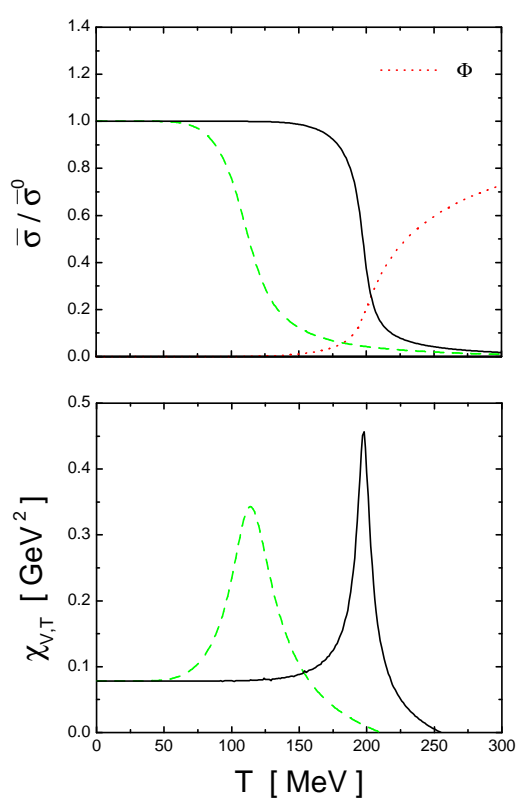

FIG. 7: Behavior of $\bar{\sigma}$ (up) and the susceptibility $\chi_{V, T}$ (down). Solid (dashed) lines correspond to the model with (without) the coupling to the Polyakov loop. In the upper panel, the dotted line shows the behavior of the traced Polyakov loop $\bar{\Phi}$.

tial for a given set of model parameters. In addition, we are interested in the chiral susceptibility $\chi_{V, T}$, which can be used to determine the chiral phase transition temperature. In Fig. 7 we show the corresponding numerical results for the analysis performed in Ref. [18], where we have considered a model based in Scheme I extended to three flavors. The results are qualitatively similar either if one considers nonlocal interactions based on Scheme II, or a two-flavor model [33]. In the upper panel we show the behavior of the mean field value $\bar{\sigma}$, normalized to its value at $T=0$, both for the model with and without the Polyakov loop (solid and dashed lines, respectively). It is clearly seen that in both cases the SU(2) chiral restoration proceeds as a smooth crossover, whereas there is an enhancement in the corresponding critical temperature when the effect of the Polyakov loop is taken into account. The critical values can be obtained from the peaks in $\chi_{V, T}$ shown in the lower panel of the figure. It is seen that the effect increases the critical temperature by about $80 \mathrm{MeV}$, bringing it to a better agreement with recent lattice values $T_{C}^{0(\text { Lat })}=160-200 \mathrm{MeV}$ [25]. In addition, it is seen that the inclusion of the Polyakov loop leads to an enhancement of the sharpness of the peak.

Finally, in the upper panel of Fig. 7 we also show the behavior of the mean field value of the traced Polyakov loop $\bar{\Phi}$. The latter can be interpreted as an order parameter of the deconfinement transition, $\bar{\Phi}=0$ and $\bar{\Phi}=1$ being related to confined and deconfined quarks, respectively. From the figure it is seen that the model offers an adequate description of this transition. It is found that the latter goes smoother than the chiral restoration, while both transitions are found to occur at the same temperature region, located at about $T=200 \mathrm{MeV}$. 


\section{SUMMARY}

We have discussed different features of chiral quark models that include nonlocal covariant interactions. These approaches represent natural extensions of the NJL model, and can be regarded as a step towards a more proper effective description of the underlying QCD theory. We have presented two alternative ways of introducing the nonlocality, called Schemes I and II. After performing a bosonization of the theory, we have derived analytical expressions to obtain the values of the pion mass and decay constant in terms of the model parameters $\bar{m}, G_{S}$ and $\Lambda$. For both schemes, well-known low energy relations are shown to be satisfied in the chiral limit. Considering Gaussian and Lorentzian nonlocal form factors, we have numerically determined the model parameters in order to reproduce the observed values of $m_{\pi}$ and $f_{\pi}$ together with a phenomenologically adequate value of the chiral condensate. From these analysis it is found that the more acceptable results correspond to the so-called Scheme II with a soft form factor.

In the framework of these nonlocal models we have analyzed the quark matter phase diagram. First we have considered isospin symmetric matter at finite temperature and baryon chemical potential, including quark-quark and quarkantiquark four-point nonlocal interactions. The ratio between the involved coupling constants has been fixed to be $H / G_{S}=$ 0.75 , as suggested by various effective approaches of the underlying quark interactions. We have derived the corresponding thermodynamical potential at the mean field level, obtaining gap equations for the mean field values of the meson and diquark fields. From a numerical analysis we have found the presence of CSB, NQM and 2SC phases, determining the characteristics of the corresponding phase transitions. This has been done for two definite model parameter sets corresponding to Schemes I and II, analyzing the dependence on the values of the ratio $H / G_{S}$ and the chiral condensate. Then we have analyzed the quark matter phase diagram under compact star constraints, namely color and electric charge neutrality and $\beta$ equilibrium. These conditions require the introduction of color and electric charge chemical potentials $\mu_{8}$ and $\mu_{Q_{q}}$. In this case it has been found that at low temperature and intermediate densities a mixed 2SC-NQM phase may be preferred. In addition, a band of gapless 2SC phase is found to appear at the border of the superconducting region. In order to apply this approach to the physics of compact stars, we have developed a two-phase description of strongly interacting matter, in which one has a quark matter phase that is described by the previous nonlocal quark models and a nuclear matter phase that is treated within the relativistic DBHF approach. Within this framework we have found that compact stars with quark matter cores turn out to be consistent with modern observations.

Finally, we have considered an extension of the above nonlocal theories in which one takes care of the effect of gauge interactions by coupling the quarks with the Polyakov loop. Taking the traced Polyakov loop as order parameter for the confinement/deconfinement transition, it is found that for $\mu=$ 0 this transition is properly described, occurring at approximately the same temperature as the chiral restoration. Moreover, it is seen that the coupling to the Polyakov loop leads to an enhancement of the chiral restoration temperature, which brings it to a better agreement with values recently obtained in lattice calculations.

\section{Acknowledgments}

I wish to thank D.B. Blaschke, A.G. Grunfeld, and especially N.N. Scoccola for their collaboration in the research summarized here. This work has been supported in part by CONICET and ANPCyT (Argentina), under grants PIP 6009 and PICT04-03-25374.
[1] C.R. Allton et al., Phys. Rev. D 68, 014507 (2003); Phys. Rev. D 71, 054508 (2005).

[2] Z. Fodor and S.D. Katz, JHEP 0404, 050 (2004); Y. Aoki, Z. Fodor, S.D. Katz, and K.K. Szabo, JHEP 0601, 089 (2006).

[3] Y. Nambu and G. Jona-Lasinio, Phys. Rev. 122, 345 (1961); Phys. Rev. 124, 246 (1961).

[4] U. Vogl and W. Weise, Prog. Part. Nucl. Phys. 27, 195 (1991); S. P. Klevansky, Rev. Mod. Phys. 64, 649 (1992); T. Hatsuda and T. Kunihiro, Phys. Rept. 247, 221 (1994).

[5] T. Schafer and E. V. Shuryak, Rev. Mod. Phys. 70, 323 (1998).

[6] C. D. Roberts and A. G. Williams, Prog. Part. Nucl. Phys. 33, 477 (1994); C. D. Roberts and S. M. Schmidt, Prog. Part. Nucl. Phys. 45, S1 (2000).

[7] M. B. Parappilly, P. O. Bowman, U. M. Heller, D. B. Leinweber, A. G. Williams, and J. B. Zhang, Phys. Rev. D 73, 054504 (2006).

[8] E. Ruiz Arriola and L.L. Salcedo, Phys. Lett. B 450, 225 (1999).

[9] G. Ripka, Nucl. Phys. A 683, 463 (2001); R.S. Plant and M.C. Birse, Nucl. Phys. A 703, 717 (2002).

[10] I. General, D. Gomez Dumm, and N. N. Scoccola, Phys. Lett. B
506, 267 (2001); D. Gomez Dumm and N. N. Scoccola, Phys. Rev. D 65, 074021 (2002).

[11] A. Scarpettini, D. Gomez Dumm, and N. N. Scoccola, Phys. Rev. D 69, 114018 (2004)

[12] Phys. Rev. C 72, 014909 (2005).

[13] R. S. Duhau, A. G. Grunfeld, and N. N. Scoccola, Phys. Rev. D 70, 074026 (2004).

[14] D. Gomez Dumm, D.B. Blaschke, A.G. Grunfeld, and N.N. Scoccola, Phys. Rev. D 73, 114019 (2006).

[15] D. Gomez Dumm, A. G. Grunfeld, and N. N. Scoccola, Phys. Rev. D 74, 054026 (2006).

[16] D. B. Blaschke, D. Gomez Dumm, A. G. Grunfeld, T. Klahn, and N. N. Scoccola, Phys. Rev. C 75, 065804 (2007).

[17] A. G. Grunfeld, J. Berdermann, D. B. Blaschke, D. Gomez Dumm, T. Klahn, and N. N. Scoccola, Int. J. Mod. Phys. E 16, 2842 (2007).

[18] G. A. Contrera, D. Gomez Dumm, and N. N. Scoccola, Phys. Lett. B 661, 113 (2008).

[19] G. Ripka, Quarks bound by chiral fields (Oxford University Press, Oxford, 1997). 
[20] M. Buballa and S. Krewald, Phys. Lett. B 294, 19 (1992).

[21] R. D. Bowler and M. C. Birse, Nucl. Phys. A 582, 655 (1995); R. S. Plant and M. C. Birse, Nucl. Phys. A 628, 607 (1998).

[22] H. Ito, W. Buck, and F. Gross, Phys. Rev. C 43, 2483 (1991); H. Ito, W. W. Buck, and F. Gross, Phys. Rev. C 451918 (1992).

[23] S. M. Schmidt, D. Blaschke, and Y. L. Kalinovsky, Phys. Rev. C 50435 (1994).

[24] M. Stingl, Phys. Rev. D 34, 3863 (1986) [Erratum-ibid. D 36, 651 (1987)]; H. J. Munczek, Phys. Lett. B 175, 215 (1986); C. J. Burden, C. D. Roberts, and A. G. Williams, Phys. Lett. B 285, 347 (1992); G. Krein, C. D. Roberts, and A. G. Williams, Int. J. Mod. Phys. A 7, 5607 (1992); D. Blaschke, G. Burau, Y. L. Kalinovsky, P. Maris, and P. C. Tandy, Int. J. Mod. Phys. A 16, 2267 (2001).

[25] C. Bernard et al. [MILC Collaboration], Phys. Rev. D 71, 034504 (2005); M. Cheng et al., Phys. Rev. D 74, 054507
(2006); Y. Aoki, Z. Fodor, S. D. Katz and K. K. Szabo, Phys. Lett. B 643, 46 (2006).

[26] M. Buballa, Phys. Rept. 407, 205 (2005).

[27] D. N. Aguilera, D. Blaschke, and H. Grigorian, Nucl. Phys. A 757, 527 (2005).

[28] M. Alford and K. Rajagopal, JHEP 0206, 031 (2002).

[29] I. Shovkovy and M. Huang, Phys. Lett. B 564, 205 (2003).

[30] T. Klähn et al., Phys. Rev. C 74, 035802 (2006).

[31] A. Dumitru, R. D. Pisarski and D. Zschiesche, Phys. Rev. D 72, 065008 (2005).

[32] S. Roessner, C. Ratti and W. Weise, Phys. Rev. D 75, 034007 (2007).

[33] D. Blaschke, M. Buballa, A. E. Radzhabov and M. K. Volkov, arXiv:0705.0384 [hep-ph]; 